Artículo

\title{
Plataforma geoespacial en línea para la recaudación de la cuota de riego
}

José Gabriel Martínez Miramontes ${ }^{1}$

Enrique Mejía Sáenz ${ }^{1 \S}$

Jesus Chávez Morales ${ }^{1,2}$

Jorge Arturo Salgado Tránsito ${ }^{2}$

${ }^{1}$ Colegio de Postgraduados-Programa de Hidrociencias y Especialistas en Recursos Naturales. Carretera México-Texcoco km 35.5, Montecillo, Texcoco, Estado de México, México. CP. 56230. (martinez.josegabriel@colpos.mx; chavezje@colpos.mx). ${ }^{2}$ Colegio Mexicano de las Flores núm. 8, s/n, Col. San Luis Huexotla, Texcoco, Estado de México, México. CP. 56220. (arturo.transito@gmail.com).

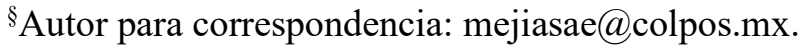

\section{Resumen}

En México, se tiene una superficie de 6.5 millones de hectáreas bajo riego, conformadas por 3.3 millones correspondientes a 86 distritos de riego y los 3.2 millones restantes a más de 40 mil unidades de riego. En virtud de que en los distritos de riego del país la posesión de la tierra y los usos del suelo y del agua son dinámicos, se requiere de tener una actualización continua de sus padrones de usuarios. Los modelos de sistema de información geográfica, a nivel de parcela de los distritos de riego de México constituyen una valiosa herramienta que necesitan ser capitalizados en el uso de diferentes aplicaciones para mejorar el desarrollo de las actividades que involucran la atención de dichos distritos. En este trabajo, partiendo de la actualización del padrón de usuarios y del catastro en el modelo de SIG del módulo 5 Tepatepec del Distrito de Riego 03 Tula, Hidalgo, se integra el sistema de recaudación de la cuota por servicio de riego en una plataforma geoespacial en línea. Esto permitirá obtener un padrón de usuarios, catastro, superficies cultivadas y patrón de cultivos en tiempo real, actualizados a través del proceso de cobro de la cuota por servicio de riego.

Palabras clave: padrón de usuarios, sistemas de información geográfica, tiempo real.

Recibido: junio de 2020

Aceptado: septiembre de 2020 


\section{Introducción}

La legislación de las primeras décadas del siglo XX propuso una política de entrega de los distritos de riego a los usuarios; política que, en los hechos, se llevó a cabo como lo demuestran en cuatro casos analizados (Distrito de Riego 01 Pabellón, Aguscalientes, 027 Ixmiquilpan, Hidalgo, módulo I primera unidad del Distrito de Riego 09 Valle de Juárez, Chihuahua y el módulo V Santa Rosa del Distrito de Riego 034 estado de Zacatecas). La organización autogestiva de los usuarios tuvo continuidad hasta la transferencia y después de ésta. La administración por los usuarios de los distritos entregados tuvo continuidad hasta la legislación y política de transferencia de la administración del estado a los usuarios en la década de 1990 (Rodríguez y Palerm, 2007).

La Comisión Nacional del Agua creada en 1989, inició un proceso de transferencia de manejo, operación, conservación y mantenimiento de áreas territoriales de los distritos de riego, definidas como módulos de riego (llamados genéricamente módulos). Los usuarios de dichos módulos se organizaron para su administración, en asociaciones civiles de usuarios (ACU), sin fines de lucro, a las que se concesionó el uso y gestión del agua de riego. Para 1996, 372 asociaciones civiles de usuarios de riego habían sido formadas para controlar las entregas de agua para más de 2.92 millones de hectáreas (Arredondo y Wilson, 2005).

Actualmente, esta transferencia está prácticamente terminada y la gestión y administración de los módulos de los distritos de riego está a cargo de sus usuarios, de acuerdo al esquema operacional indicado, bajo la estrecha supervisión de la Comisión Nacional del Agua (CNA), Velasco et al. (1994), menciona que el padrón de usuarios es uno de los documentos fundamentales de un distrito de riego, del que derivan los programas y acciones que éste realiza, y con base en el cual se establecen las dimensiones de los presupuestos, costos, cuotas, tiempos de ejecución, valor y volumen de producción y de agua, superficies, cultivos, etc.

En 1969, en el Distrito de Riego 038 Río Mayo, Sonora, se examinó la factibilidad de la aplicación del cómputo electrónico a la solución de problemas de cálculo rutinario en diversas áreas de trabajo de distritos de riego (Chávez,1974). Actualmente, en los distritos de riego del país se tiene en operación el 'sistema de padrón de usuarios' en su versión 3, llamado SIPAD 3 (Gerencia de Distritos de Riego, 2004). En los distritos de riego del país la posesión de la tierra y los usos del suelo y del agua son dinámicos, por lo que se requiere una actualización continua de sus padrones de usuarios.

La Ley de Aguas Nacionales (LAN) publicada en diciembre de 1992, en su título cuarto, capítulo iv, artículo 30, fracción 8, menciona que 'la Comisión Nacional del Agua en el ámbito nacional y los organismos de cuenca en el ámbito de las regiones hidrológico-administrativas, llevarán el registro público de derechos de agua en el que se inscribirán los padrones de usuarios de los distritos de riego, debidamente actualizados' (LAN, 1992). Bajo este contexto, donde la LAN mandata el tener los Padrones de Usuarios actualizados, es necesario el utilizar las herramientas de frontera que permitan esta actualización. Estas herramientas son los modelos de Sistema de Información Geográfica (SIG), las aplicaciones en línea vía internet y los sistemas automatizados de recaudación de la cuota por servicio de riego. 
Un SIG es un conjunto de herramientas que permiten el procesamiento de información de datos de tipo espacial usados para tomar decisiones acerca de cierto espacio o área específica de la tierra o también para visualizar de forma general un espacio de acuerdo al contenido de bases de datos digital (Sosa y Martínez, 2009). De la misma manera, Otaya et al. (2006), los definen como una herramienta de hardware, software y procedimientos diseñados para facilitar la obtención, gestión, manipulación, análisis, modelado y salida de datos espacialmente referenciados, para resolver problemas complejos de planificación y gestión.

De acuerdo a Mejía et al. (2003), el desarrollo de un SIG para un distrito de riego y los módulos que lo integran puede permitir, de una manera rápida y confiable: a) disponer, procesar y analizar la información geográfico-estadística generada durante el desarrollo de las actividades que hacen posible el funcionamiento del distrito de riego; b) actualizar el padrón de usuarios; c) actualizar el inventario de infraestructura hidroagrícola; d) integrar el SIG generado, con el sistema de recaudación del distrito de riego; y e) integrar el SIG con la información climática y de operación para la toma de decisiones en tiempo real.

Arnab y Santanu (2018), menciona que la generación actual de SIG comercial no puede facilitar la toma de decisiones en tiempo real sin modificaciones significativas o integración con modelos externos. En general, la tecnología de las geografías digitales ha encontrado que la representación del cambio en el tiempo es extremadamente difícil de manejar.

El SIG hoy en día sigue siendo una tecnología para datos estáticos, que es un impedimento importante para su uso en el modelado espacial Palacios et al. (2002), mencionan que, para lograr el desarrollo sostenible de la agricultura de riego, es necesario que las asociaciones civiles de usuarios (ACUs) establezcan cuotas de cobro por servicio de riego que les permitan ser autosuficientes económicamente, debido a que el precio del agua de riego puede alcanzar un importante número de metas sociales y de manejo.

Según (Palacios y Exebio, 1989) citado por Santos et al. (2000), en los distritos de riego de México existen diferencias en cuanto al costo del servicio de riego y la forma de recuperarlo; básicamente hay dos formas de cobro: por superficie regada o por volumen utilizado. Una modalidad intermedia es por hectárea por riego, donde el pago del servicio es función del número de riegos efectuados en la unidad de superficie durante un ciclo agrícola.

En la actualidad, se han creado diversas herramientas tecnológicas, software y hardware, que integradas permitan facilitar la gestión técnica y administrativa en los distritos de riego Mundo y Martínez (2002), crearon un sistema computarizado para la gestión del agua en sistemas de riego por gravedad en México (SICODE) el cual es un sistema de cómputo que integra de manera modular los siguientes programas: base de datos, sistema de información geográfica, simulación de balance de agua en el suelo (pronóstico del riego, apoyado en estaciones meteorológicas automatizadas), simulador biológico y sistema experto. Fue implementado en el distrito de riego: 010 Culiacán en el estado de Sinaloa, México.

Sagols et al. (2007), desarrollaron el sistema SIGTERNET, la cual es una herramienta para construir, mantener y consultar Sistemas de Información Geográfica (SIG) bajo una arquitectura cliente-servidor accesible vía internet, Delipetrev et al. (2014) desarrollaron un prototipo de aplicación web para recursos hídricos utilizando los últimos avances en tecnologías de la 
información y la comunicación (TIC), software de código abierto y GIS web. La aplicación web tiene tres servicios web para: 1) gestión, presentación y almacenamiento de datos geoespaciales; 2) soporte de modelado de recursos hídricos; y 3) optimización de recursos hídricos.

Rueda et al. (2018), diseñaron colaborativamente una plataforma Web-SIG con una interface y contenidos correspondientes a indicadores espaciales capaces de generar datos que revelen el grado de naturalidad, biodiversidad y el efecto de los humedales urbanos. En México, durante los años 2006 a 2012 se realizaron modelos de SIG a nivel de parcela para 85 distritos de riego. Este fue un gran esfuerzo como Nación para generar una plataforma geográfica única a nivel mundial. Sin embargo, no se ha cristalizado en aplicaciones que permitan un mejor manejo de los distritos y módulos de riego.

Actualmente, en todos los distritos de riego del país, el padrón de usuarios y planos catastrales no están actualizados, lo cual implica que los sistemas de cobro de la cuota por servicio de riego no corresponden a la realidad, con los conflictos administrativos que esto conlleva. Asimismo, se tienen tres 'padrones' en cada distrito: 1) el padrón de usuarios oficial que tiene la CONAGUA; 2) el padrón de usuarios que tiene la ACU del módulo de riego; y 3) el padrón de usuarios con el que se cobra la cuota por servicio de riego.

La coexistencia de estos tres padrones implica una seria problemática para el adecuado manejo de los distritos y módulos de riego. Adicionalmente, el reporte de las estadísticas agrícolas en los distritos y módulos de riego presenta serias inconsistencias al no tener el personal técnico suficiente encargado de estas actividades.

En este trabajo, se desarrolla la implementación de una plataforma geoespacial en línea para la recaudación de la cuota por servicio de riego, donde se integran las herramientas de frontera para la actualización del padrón de usuarios, planos catastrales, así como las estadísticas agrícolas, lo cual permitirá mejorar el proceso de administración del módulo 5 Tepatepec del Distrito de Riego 03 Tula, Hidalgo, bajo la siguiente hipótesis: es posible obtener la actualización del padrón de usuarios, planos catastrales y estadísticas agrícolas en tiempo real a través de la recaudación de la cuota por servicio de riego si se apoya con la implementación de una plataforma geoespacial en línea.

\section{Materiales y métodos}

Los trabajos se realizaron en el Módulo de Riego No. 5 denominado 'usuarios y productores unidad Tepatepec, AC' del Distrito de Riego 003 Tula, Hidalgo. Se localiza entre los paralelos 20 $12^{\prime}$ y $20^{\circ} 15^{\prime}$ de latitud norte y los meridianos $99^{\circ} 01^{\prime}$ y $99^{\circ} 08^{\prime}$ de longitud oeste. En la Figura 1, se presenta la localización del Módulo de Riego No. 5 'usuarios y productores unidad Tepatepec, AC, en el estado de Hidalgo. 


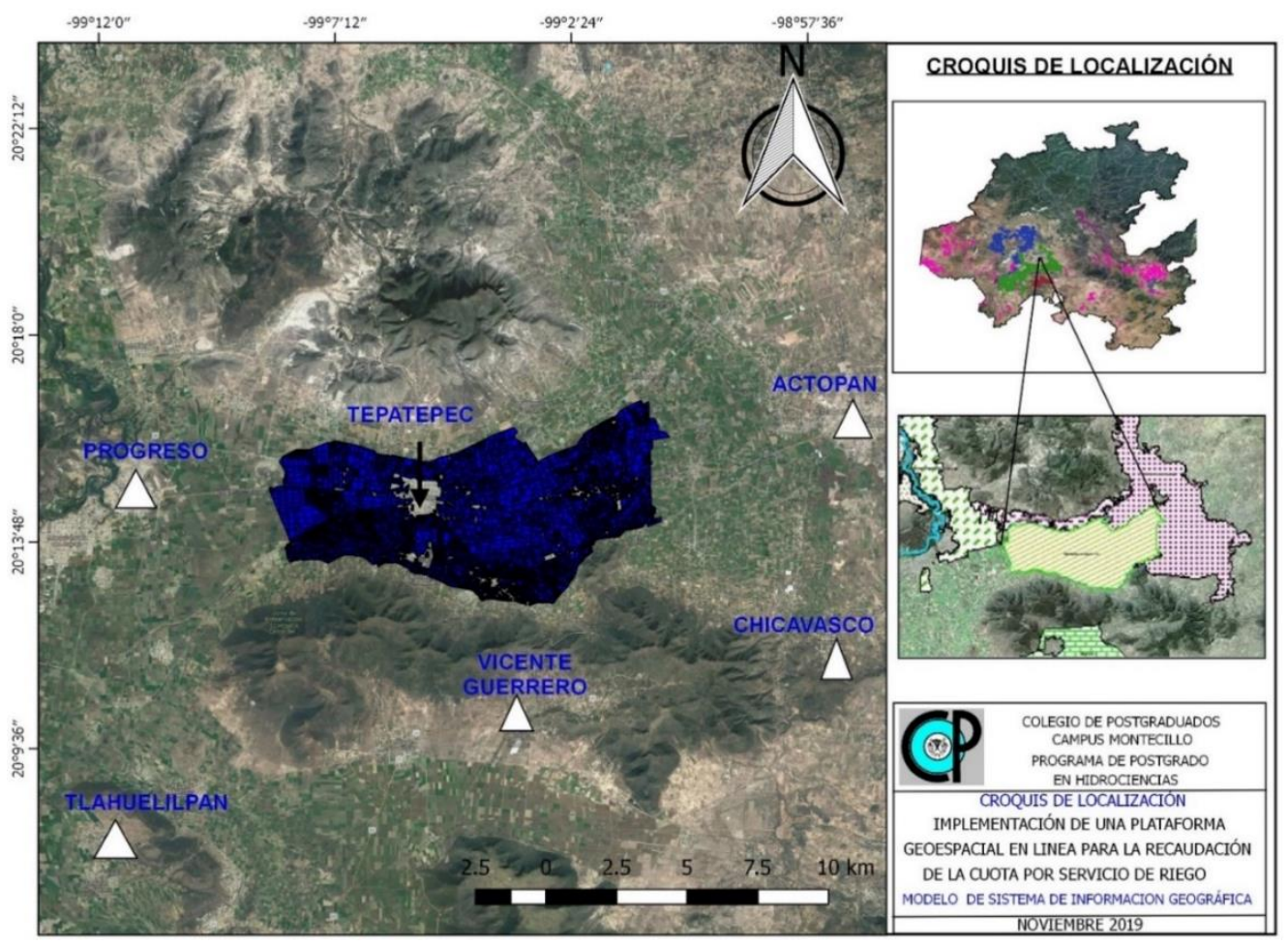

Figura 1. Localización del área de estudio.

Para llevar a cabo el presente trabajo, se desarrolló la metodología que se muestra en la Figura 2.

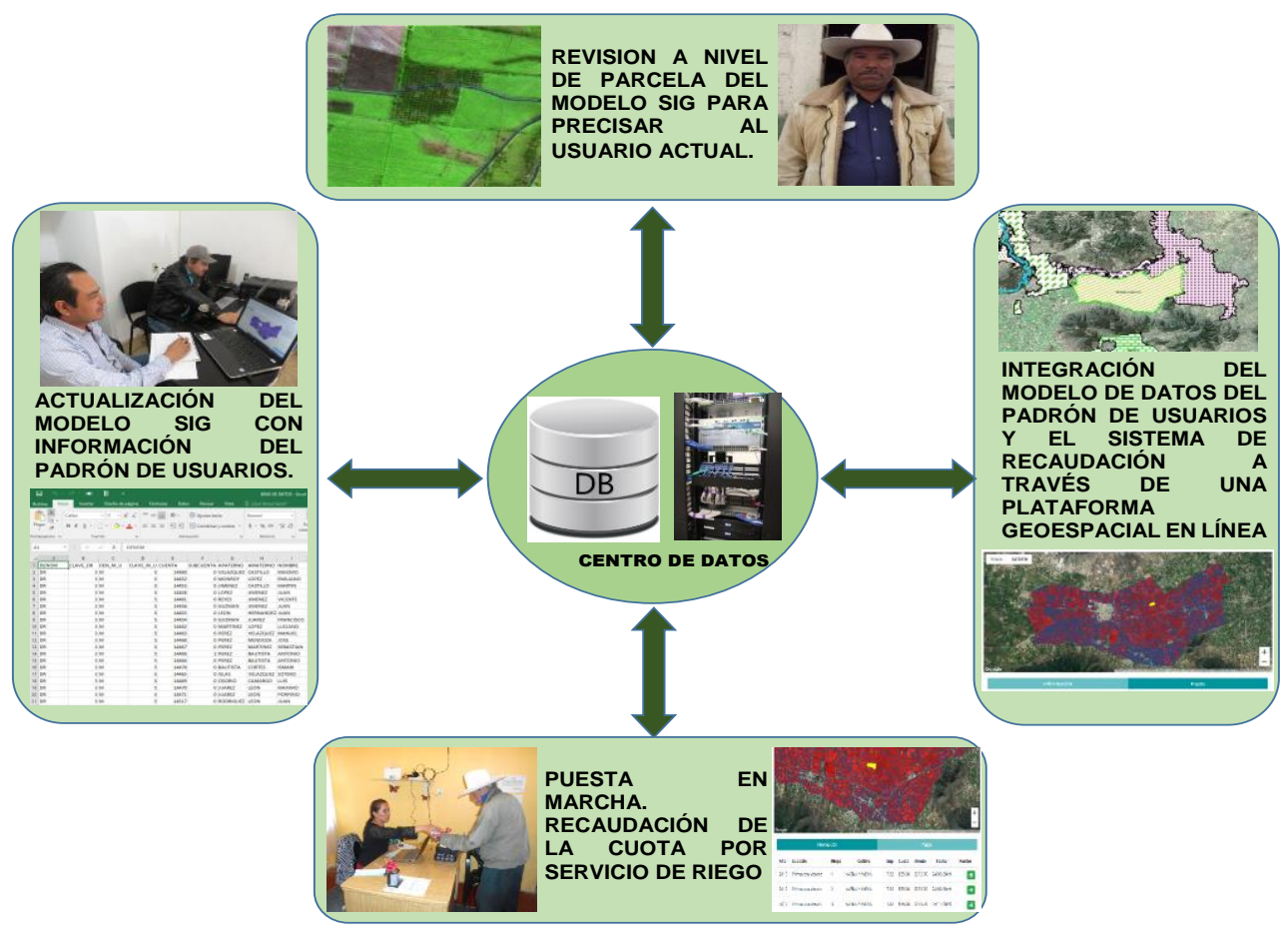

Figura 2. Metodología desarrollada en el presente trabajo. 
A continuación, se presenta la descripción de cada una de las etapas.

\section{Revisión a nivel de parcela del modelo de SIG para precisar al usuario actual}

En una primera revisión, se obtuvieron los cambios referentes a superficie de riego, ubicación de la parcela y nombre de usuario entre la base de datos del padrón de usuarios del distrito, módulo de riego y la base de datos del modelo de SIG mediante el número de cuenta. Las cuentas que eran consistentes en su información se dieron por válidas. Posteriormente, las cuentas en las cuales había información inconsistente en sólo algún dato o que no estuvieran en cualquiera de las tres bases de datos, se procedió a su revisión en campo. Dichas parcelas se localizaron en campo para recabar la información correcta y actualizada durante los meses de enero a junio del 2019.

\section{Actualización del modelo de SIG con la información del padrón de usuarios}

Se identificaron los requerimientos de actualización del modelo de SIG, con base a las cuentas del padrón de usuarios proporcionado y la información recabada en campo a nivel de parcela. En el Cuadro 1, se presenta la clasificación de las necesidades de actualización del padrón de usuarios de la siguiente manera.

Cuadro 1. Clasificación de las necesidades de actualización del padrón de usuarios.

Tipo de actualización Características de las cuentas

Cambio de usuario $\quad$ Son aquellas parcelas en donde el propietario ya no es el que se indica en la base de datos del padrón de usuarios del modelo de sistema de información geográfica.

Cambio de usuario y Son aquellas parcelas en donde tanto el propietario y la superficie superficie sufrieron cambios por lo que se requirió realizar la actualización.

Cambio de superficie Se refiere a las parcelas donde el propietario es el mismo, pero se requirió actualizar la superficie.

Cuentas y subcuentas Son aquellas parcelas que se encuentran en la base de datos del padrón creadas de usuarios, sin embargo, aún no habían sido dados de alta en el modelo de sistema de información geográfica.

Sin cambios

En estas parcelas la información recabada en campo y gabinete no sufren cambios.

Por último, se actualizó el modelo de SIG con la información de los cambios respectivos tanto en la base de datos del Padrón de Usuarios y en el parcelamiento (shapefile) según haya sido el caso.

\section{Integración del modelo de datos del padrón de usuarios y el sistema de recaudación a través de una plataforma geoespacial en línea}

La plataforma geoespacial en línea significa que el modelo de SIG y el sistema de recaudación sean accesibles a través de Internet. Para lograrlo, se siguieron dos etapas, las cuales se describen a continuación: En una primera etapa, partimos de la base de datos, la cual es el componente esencial del modelo de SIG porque en ella se organiza y almacena el contenido (vectores 'shp' y tablas 
'dbf') de las aplicaciones que forman parte de la plataforma geoespacial, en este caso, el sistema de recaudación. Para esto, es vital visualizar los flujos de los geoprocesamientos que ilustren el escenario idóneo para la ejecución de la base de datos a partir del modelo conceptual, lógico y físico.

De esta forma, los sistemas gestores de base de datos son la herramienta más adecuada, ya que permite el almacenamiento y la manipulación de objetos geométricos vectoriales, procesamiento de transacciones, integridad referencial, procedimientos almacenados y estandarización del lenguaje de consulta y funcionalidad avanzada (Bustos, 2012).

Para realizar el diseño de la base de datos espacial se siguieron los siguientes pasos: se definieron los requerimientos funcionales del sistema de recaudación de la cuota por servicio de riego. Diseño conceptual: para representar la información geométrica y topológica se usó el modelo basado en entidades, el cual concibe objetos geográficos embebidos en el espacio. En un objeto geográfico se distinguen dos componentes: 1) una descripción; y 2) una componente espacial, que corresponde con la forma y ubicación del objeto en el espacio.

Esta vista de la información geográfica reúne dentro de un objeto espacial puntos del espacio subyacente que comparten propiedades similares, esto es, tienen la misma descripción. Para poder distinguir a un objeto de otros, a cada objeto se le asigna una identificación. El conjunto entidad completo (identificación, objeto espacial y descripción común constituye un objeto geográfico.

\section{Diseño lógico (mapeo de modelo de datos)}

Se implementa la base de datos en PostgreSQL; a partir, del mapeo del modelo basado en entidades y usando el modelo de datos especifico del sistema gestor de base de datos (modelo de datos de implementación), para este caso se usó el modelo relacional.

\section{Diseño físico}

Se especifica la estructura de almacenamiento de los archivos, su organización, índices, y rutas de acceso. Simultáneamente, se diseñan e implementan los programas de aplicación correspondientes a las transacciones, para este caso el sistema de recaudación. La etapa 2 depende, directamente, de la estructura del repositorio de datos creado en la primera etapa, debidamente documentado, en el que se dicta las pautas y lineamientos a seguir para presentar los productos en una plataforma geoespacial en línea.

Según (Olaya, 2011) citado por (Núñez, 2014), el avance de las redes locales y de Internet 'ha permitido que se acceda a la información geográfica contenida en un SIG utilizando el paradigma cliente-servidor. Para ello es necesario contar con componentes en el lado servidor que distribuyan la información y componentes en el lado del cliente para acceder a esta'. En la Figura 3, podemos observar de manera general la arquitectura simple para un sitio web dinámico, como es el caso del presente trabajo. 


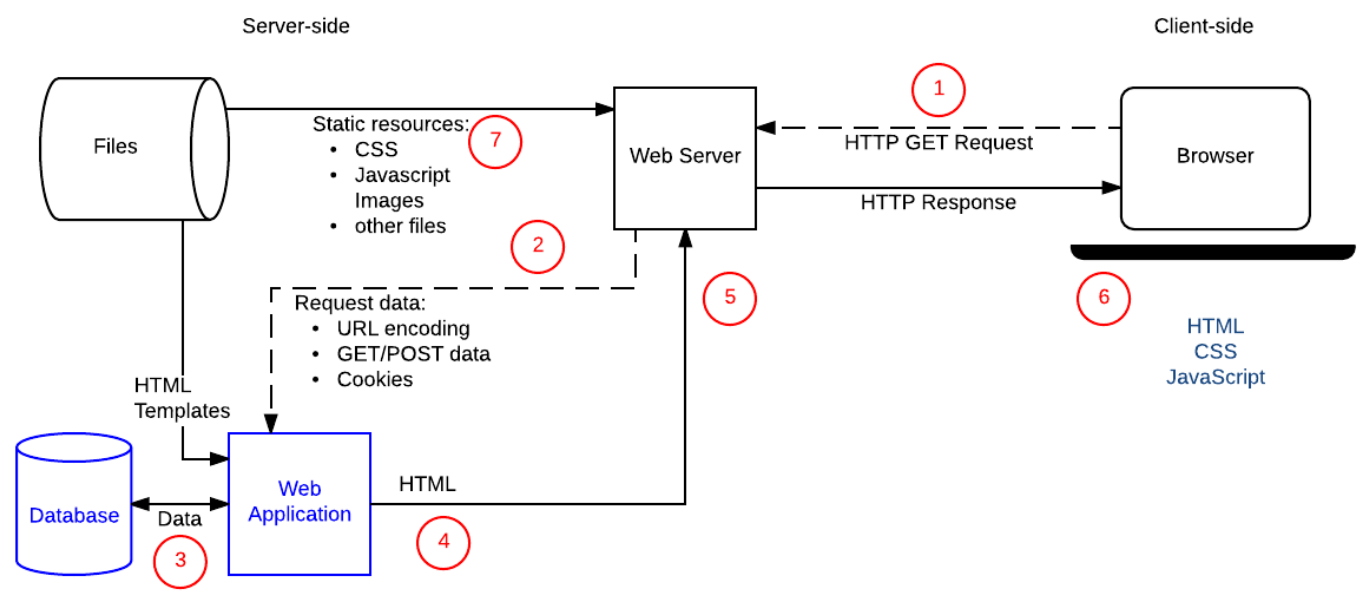

Figura 3. Arquitectura simple de un sitio web dinámico (www.developer.mozilla.org).

De la misma manera, la plataforma geoespacial en línea para la recaudación de la cuota por servicio de riego estará ligado a la base de datos espaciales a través de la aplicación web GeoServer y GeoExplorer, ambas herramientas agrupadas en el Web Server.

\section{Puesta en marcha. Recaudación de la cuota por servicio de riego}

Una vez que tenemos generada la plataforma geoespacial en línea procedemos a realizar la puesta en marcha de esta. En el módulo, su cuenta con cuatro oficinas recaudadoras dispuestas para atender las cinco secciones de riego. Durante el mes de septiembre de 2019, se explicó al personal de cada oficina recaudadora el funcionamiento de la plataforma geoespacial para poder realizar el cobro de la cuota por servicio de riego. En la Figura 4, se presenta el proceso a seguir para realizar la recaudación de la cuota por servicio de riego.

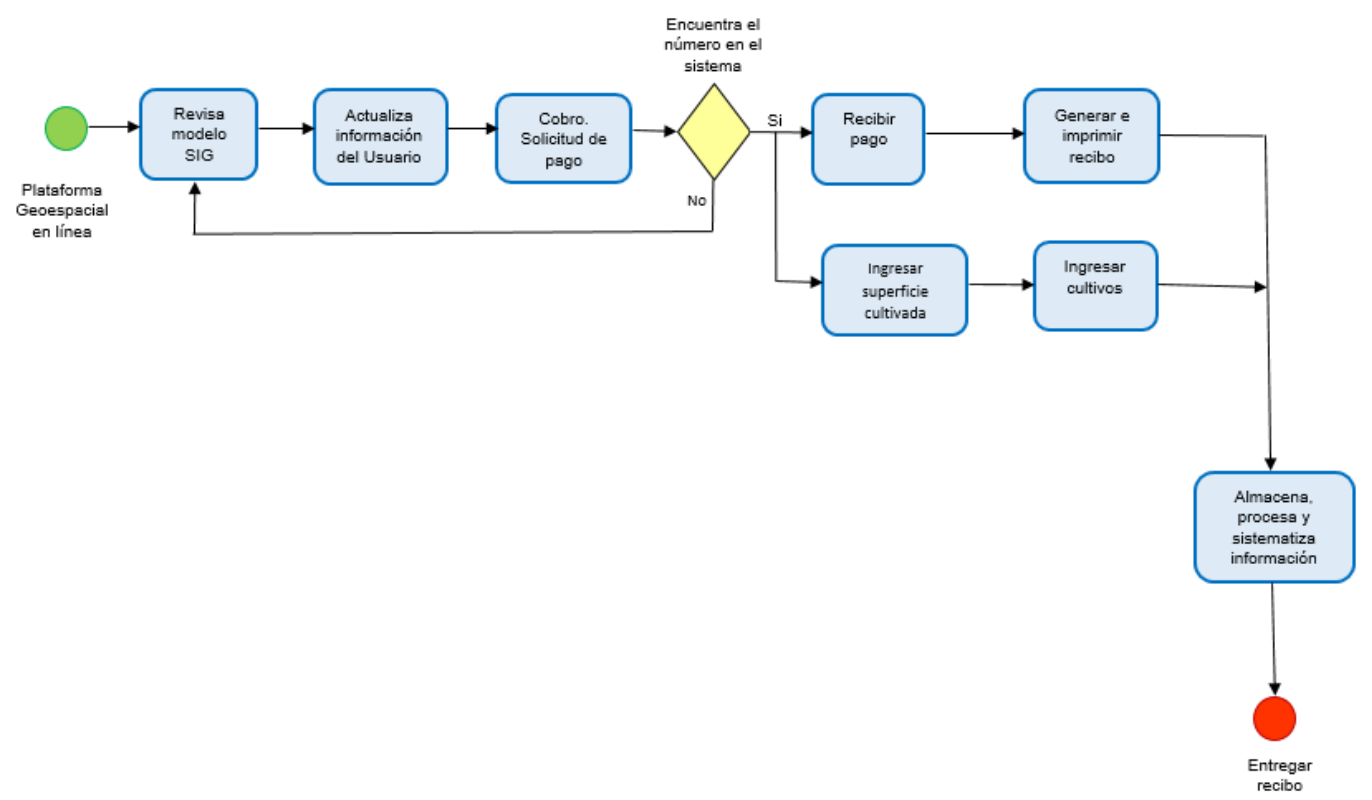

Figura 4. Proceso de recaudación de la cuota por servicio de riego. 
La finalidad de la puesta en marcha consistió en probar el proceso de recaudación implementado para detectar posibles inconsistencias entre la relación de las entidades geográficas, tabla de atributos y tabla de cobros. Se instruyó al personal para que en el caso en que no se encuentre el número de cuenta en la plataforma geoespacial se nos notificara para revisar y actualizar el padrón de usuarios. Siguiendo el proceso de recaudación, se ingresa la información necesaria para poder recibir el pago y posteriormente generar e imprimir el recibo el cual se le entrega al usuario. La información generada en el proceso de cobro queda almacenada en la plataforma geoespacial en línea para obtener el Padrón de Usuarios actualizado, planos catastrales e información estadística agrícola en tiempo real.

\section{Resultados}

\section{Revisión y actualización del modelo de SIG}

Se revisó y actualizó el modelo de SIG correspondiente a la información de la base de datos del padrón de usuarios generada y las entidades geográficas. En el Cuadro 2, se presenta el número de cuentas revisadas y actualizadas.

Cuadro 2. Revisión y actualización del padrón de usuarios.

\begin{tabular}{cccc}
\hline Tipo de actualización & Número de cuentas & $(\%)$ & Superficie (ha) \\
\hline Sin cambios & 3131 & 62.1 & 3355.57 \\
Cambio de usuario & 1488 & 29.51 & 1646.83 \\
Cuentas y subcuentas creadas & 261 & 5.18 & 178.81 \\
Cambio de superficie & 104 & 2.06 & 100.72 \\
Cambio de usuario y superficie & 58 & 1.15 & 68.29 \\
Total & 5042 & 100 & 5350.22 \\
\hline
\end{tabular}

La base de datos del padrón de usuarios asciende a 5350.22 ha y 5042 cuentas de las cuales, se actualizó 37.9\% del padrón de usuarios correspondiente a 1911 cuentas. Los resultados de la revisión y actualización de las cuentas del padrón de usuarios se pueden observar en la Figura 5.

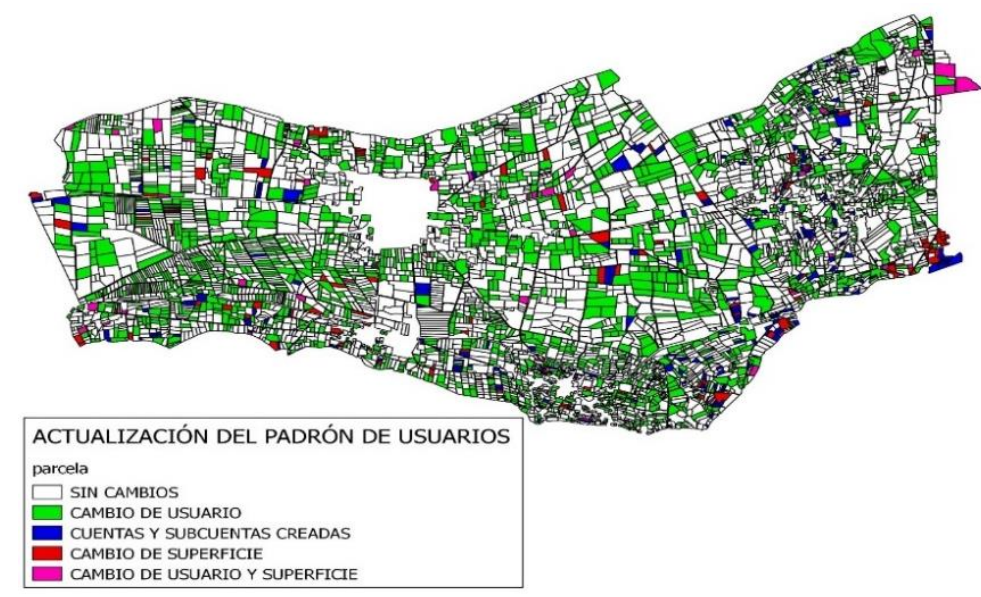

Figura 5. Representación gráfica de la revisión y actualización del padrón de usuarios. 


\section{Integración del modelo de datos del padrón de usuarios y el sistema de recaudación a través de una plataforma geoespacial en línea}

Se procedió a la implementación del modelo de base de datos del padrón de usuarios en el motor de datos PostgreSQL, con la capacidad de gestionar datos desde QGIS. Una vez que es probado el repositorio de datos, se inicia la implementación de la plataforma geoespacial en línea. En esta etapa se desarrolla la conexión con la base de datos, la edición de los datos geográficos a subir a la red y la inserción dentro de un ambiente web geográfico para la visualización en internet. Se utiliza en esta actividad, las herramientas GeoServer y GeoExplorer. De forma resumida, la primera es un servidor de mapas para compartir, analizar y editar datos geoespaciales. Mientras que la segunda es una aplicación para composición, estilo, edición y publicación de mapas desde el navegador.

Para la incorporación del modelo SIG y el sistema de recaudación en internet se utiliza GeoExplorer, que contiene las herramientas necesarias para enganchar los datos de forma directa a la web, sin procesos de programación o instructivos de implementación. La secuencia para construir la plataforma geoespacial con el modelo de SIG y el sistema de recaudación se explica de la siguiente manera: a) la plataforma GeoExplorer debe conectarse al servidor de mapas GeoServer; b) se añade la capa al mapa y se conecta al GeoServer Local dando el visto bueno para la publicación; c) si se quieren realizar modificaciones de estilo, etiquetas, colores con la herramienta de estilo dentro la misma página se realizan los cambios; y d) como producto se obtiene una plataforma geoespacial que incluye, además del modelo de SIG y el sistema de recaudación, coberturas externas de googlemaps y openstreet maps, herramienta de acercamiento, consulta, identificación, entre otras.

Una vez desarrollado el modelo de SIG y el sistema de recaudación, se construye un portal Web que sirve como plataforma geoespacial en línea para realizar el proceso de cobro de la cuota por servicio de riego y consulta de información. Para tener un mayor control en el acceso a la información, se creó un formulario de autentificación, en el cual es necesario contar con el nombre de usuario y el password correspondiente. Posteriormente, se puede observar un visualizador en donde se muestra el modelo de SIG representado por entidades geográficas (polígonos). De fondo se puede observar la imagen satelital obtenida desde el servidor de Google.

Para poder seleccionar una entidad geográfica (parcela), se da click sobre el centro del polígono y se pintará de color amarillo, al mismo tiempo que seleccionamos la entidad geográfica, en la parte inferior, aparece la información cargada en la plataforma geoespacial en línea, tal como, datos del productor, datos del cultivo, red de canales y otros datos, como se puede observar en la Figura 6. 


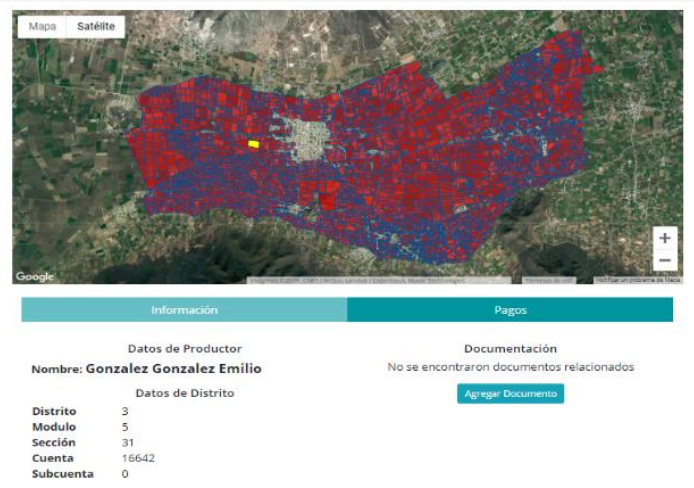

Figura 6. Plataforma geoespacial en línea.

\section{Puesta en marcha. Recaudación de la cuota por servicio de riego}

Para realizar el proceso de recaudación de la cuota por servicio de riego, se inicia seleccionando el menú de búsquedas para realizar la localización del predio. La búsqueda se puede realizar por cuenta o nombre de usuario. Posteriormente, como se puede observar en la Figura 7, seleccionamos la búsqueda del predio por cuenta y se despliega el nombre del usuario. Al dar click en seleccionar, la plataforma geoespacial nos permite visualizar la parcela al centro del modelo de SIG. Una vez que se realizó la búsqueda y ubicamos la cuenta, se procede a realizar el cobro de la cuota por servicio de riego. Al seleccionar en el menú 'pagos', nos despliega el historial de cobros y nos permite agregar un pago (Figura 8).

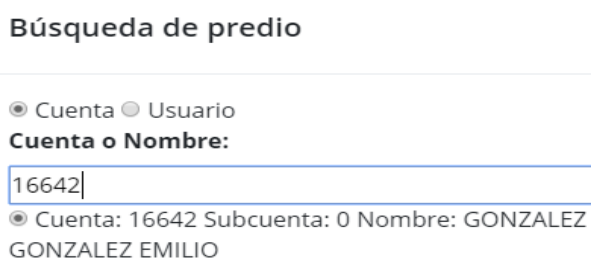

\section{Seleccionar Cerrar}

Figura 7. Selección de la búsqueda de predio por cuenta.

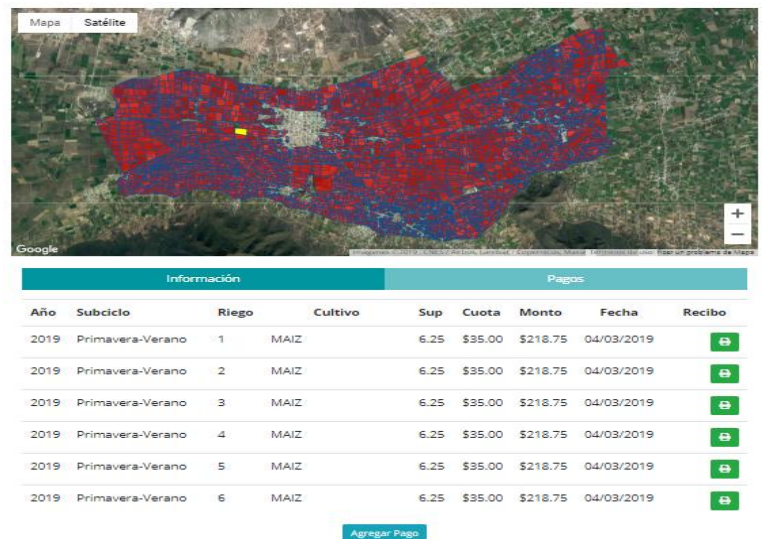

Figura 8. Visualización del proceso de cobro (agregar pago). 
Inmediatamente, se despliega una ventana emergente, en la cual para poder realizar el cobro se deben llenar los espacios indicados. Posteriormente, al dar click en la selección 'guardar', inmediatamente se despliega el historial de cobros, en donde se observa que se ha cargado el cobro que previamente se realizó (Figura 9).

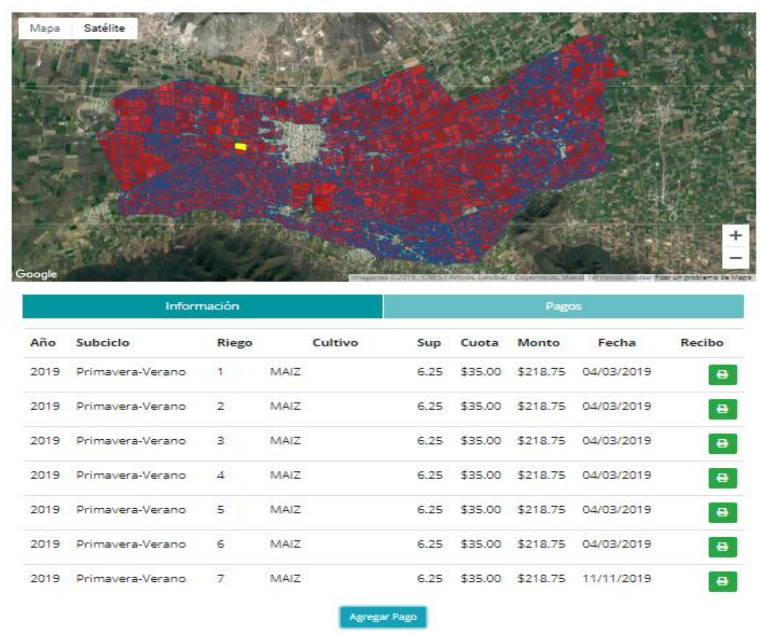

Figura 9. Historial de cobros de la cuota por servicio de riego.

Una vez agregado el pago, se procede a generar el recibo correspondiente para su impresión, como se observa en la Figura 10 y posteriormente se entrega al usuario (Figura 12). Adicionalmente, la búsqueda del predio, también se puede realizar por nombre, seleccionando la cuenta que corresponda al usuario, lo cual podemos observarlo en la Figura 11, posteriormente se sigue el mismo procedimiento descrito anteriormente. A continuación, se muestra en la Figura 12 , la puesta en marcha de la plataforma geoespacial en línea para la recaudación de la cuota por servicio de riego.

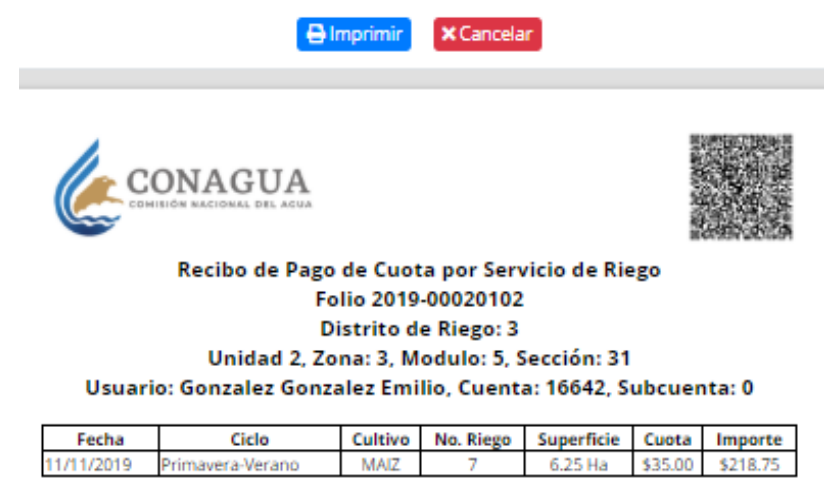

Firma del Recaudador

Figura 10. Generación del recibo de pago de la cuota por servicio de riego. 
Búsqueda de predio

C Cuenta $\odot$ Usuario

Cuenta o Nombre:

GONZALEZ GONZALEZ EMILIO

Cuenta: 16642 Subcuenta: 0 Nombre: GONZALEZ

Cuenta: 16642 Sub

- Cuenta: 16631 Subcuenta: 0 Nombre: GONZALEZ

GONZALEZ EMILIO

Cuenta: 16973 Subcuenta: 0 Nombre: GONZALEZ

GONZALEZ EMILIO

Cuenta: 16972 Subcuenta: 0 Nombre: GONZALEZ

GONZALEZ EMILIO

Figura 11. Búsqueda del predio por nombre de usuario.

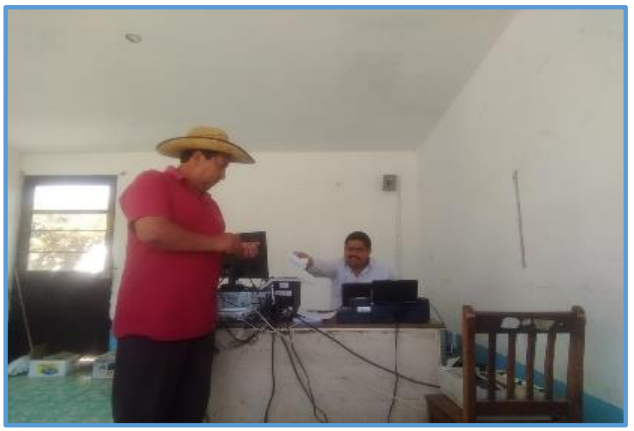

Figura 12. Puesta en marcha y entrega de recibo de pago (Oficina Tothié).

\section{Almacenamiento y manejo de la información disponible}

La plataforma geoespacial almacena la información la cual está disponible para la obtención del padrón de usuarios actualizado, planos catastrales e información estadística agrícola en tiempo real. A continuación, se muestra de manera gráfica los planos catastrales e información de patrón de cultivos obtenidos de la implementación de la plataforma geoespacial en línea (Figura 13).

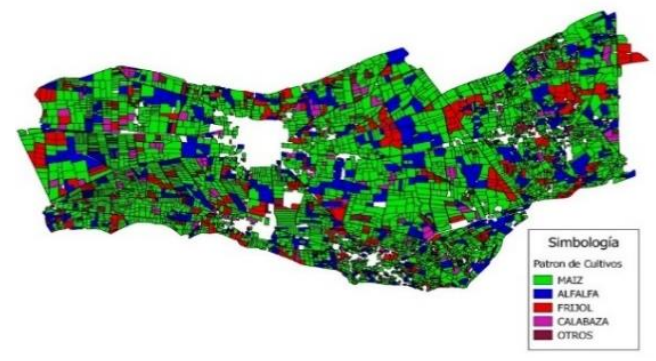

Figura 13. Patrón de cultivos.

En el Cuadro 3, se muestra la información correspondiente al patrón de cultivos con superficie cultivada derivado de la actualización del padrón de usuarios. 
Cuadro 3. Patrón de cultivos con superficie cultivada.

\begin{tabular}{ccc}
\hline Cultivos & Superficie cultivada & $(\%)$ \\
\hline Maíz & 3611.25 & 67.5 \\
Alfalfa & 936.29 & 17.5 \\
Frijol & 481.52 & 9 \\
Calabaza & 187.26 & 3.5 \\
Otros & 133.9 & 2.5 \\
Total & 5350.22 & 100 \\
\hline
\end{tabular}

El patrón de cultivos asciende a 5350.22 ha, se observa que $67.5 \%$ corresponde a maíz, $17.5 \%$ corresponde a alfalfa, $9 \%$ a frijol, $3.5 \%$ a calabaza y $2.5 \%$ a otros.

\section{Conclusiones}

De la revisión del padrón de usuarios se obtuvo que 3131 cuentas no presentan cambios, se actualizaron 1911 cuentas de las cuales: 1488 cuentas se actualizaron por cambio de usuario, 104 cuentas por cambio de superficie, 58 cuentas por cambio de usuario y superficie y se crearon 261 cuentas y subcuentas. Lo anterior, permitió tener el padrón de usuarios actualizado al mes de agosto de 2019. Se logró que el modelo de SIG y el sistema de recaudación sean accesibles a través de Internet para realizar la recaudación de la cuota por servicio de riego en una plataforma geoespacial.

La implementación de la plataforma geoespacial en línea permite tener un proceso continuo en la actualización del padrón de usuarios, planos catastrales y estadísticas agrícolas en tiempo real a través de la recaudación de la cuota por servicio de riego. Actualmente, se realiza la recaudación de la cuota por servicio de riego en el módulo 5 del Distrito de Riego 003 Tula, Hidalgo, en donde se actualiza constantemente el padrón de usuarios y se dispone de los planos catastrales al día e información de patrón de cultivos y superficies cultivadas en tiempo real.

\section{Literatura citada}

Arnab, B. and Santanu, R. 2018. Spatial models and geographic information systems. In: $2^{\text {th }}$ edicion. Visva-Bharati University. Santiniketan, India. Encyclopedia of Ecology. 1-11 pp.

Arredondo, S. M. y Wilson, P. N. 2005. Un análisis sobre la transferencia de la gestión del riego en México. México. Rev. Mex. Agroneg. 16(6):422-437.

Bustos, X. 2012. Desarrollo de un sistema de información geoespacial con uso de software libre. Venezuela. Terra Nueva Etapa. 44(28):11-38.

Chávez, J. 1974. Auxilio de computadores electrónicos en el procesamiento de la información para la operación de distritos de riego. Tesis Profesional. Escuela Nacional de Agricultura. Chapingo, Estado de México, DF. 85 p.

Comisión Nacional del Agua. 2009. Normas para la formación, actualización y sistematización del padrón de usuarios de los distritos de riego. Instructivo técnico. Primera edición. Secretaría de Medio Ambiente y Recursos Naturales. Cd. de México, México. 130 p.

Delipetrev, B.; Jonoski, A. and Solomatine, D.P. 2014. Development of a web application for water resources based on open source software. Holanda. Computers \& Geosciences. 62:35-42. 
Gerencia de Distritos de Riego. 2004. SIPAD 3. Sistema de padrón de usuarios. Manual de usuarios. Subdirección General de Infraestructura Hidroagrícola. Comisión Nacional del Agua. México.

LAN. 1992. Ley de Aguas Nacionales. Última reforma publicada DOF 24-03-2016.

Mejía, E.; Exebio, A.; Palacios, E.; Santos, A. L. y Delgadillo, M. E. 2003. Mejoramiento del manejo de distritos y módulos de riego utilizando sistemas de información geográfica. México. Terra. 4(21):513-522.

Mundo, M. D. y Martínez, P. 2002. Sistema computarizado para la gestión del agua en sistemas de riego por gravedad en México. México. Ingeniería del agua. 2(9):171-181.

Núñez, C. 2015. Implementación de un visor geoespacial en la web sobre recurso hídrico para el acuífero Jacó, Garabito, Puntarenas, 2014. Costa Rica. Rev. Geográfica de América Central. 54(1):45-64.

Otaya, L. A.; Sánhez, R. J.; Morales, L. y Botero, V. 2006. Los sistemas de información geográfica (SIG), una gran herramienta para la silvicultura urbana. Colombia. Revi. Facultad Nacional de Agronomía. 1(59):3201-3216.

Página web: www.developer.mozilla.org. Visión general cliente-servidor.

Palacios, E.; Exebio, A.; Mejía, E.; Santos, A. L. y Delgadillo, M. E. 2002. Problemas financieros de las asociaciones de usuarios y su efecto en la conservación y operación de distritos de riego. México. Terra. 20(4):505-513.

Rodríguez, B. y Palerm, J. 2007. Antes de la transferencia: La entrega de distritos de riego. México. Agricultura, Sociedad y Desarrollo. 2(4):105-125.

Rueda, I. A.; Rojas, C. A.; De La Fuente, H. E. y Virano, P.Z. 2018. Diseño colaborativo de una plataforma Sig Web de humedales urbanos del área metropolitana de Concepción (urbancost). Chile. Urbano. 21(37):32-43.

Sagols, F. D.; Navarro, J. M.; Ulloa, M.; Hernández, E. y López M. 2007. Sistema integral para construir y explotar bases de datos geográficas Vía Internet. México. Computación y Sistemas. 2(11):57-173.

Santos, A. L.; Palacios, E.; Exebio, A. y Chalita, L. E. 2000. Metodología para evaluar la distribución de costos e ingresos relacionados con el servicio de riego. México. Agrociencia. 5(34):639-649.

Sosa, J. y Martínez, F. 2009. Los sistemas de información geográfica y su aplicación en enlaces de comunicaciones. México. Científica. 1(13):27-34.

Velasco, I.; Balancán, A.; Jaimes, S. y Robles B. 1994. Sistema de información geográfica para distritos de riego. México. Ingeniería Hidráulica en México. 1(9):35-41. 